\title{
The fragmentation of the ocean: spray formation
}

\author{
Yves Couder $†$
}

Matières et Systèmes Complexes, Université Paris 7 Denis Diderot, CNRS - UMR 7057, Bâtiment Condorcet, 10 rue Alice Domon et Léonie Duquet 75205 Paris, CEDEX 13, France

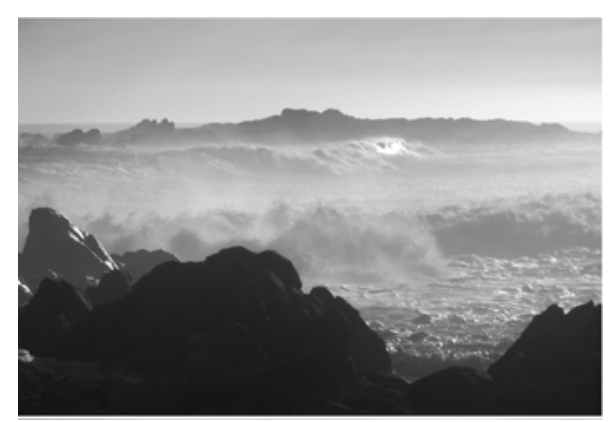

By what process can droplets be extracted out of the sea? This is an old problem, well-documented by precise field measurements of the size distribution of the spray aerosols. Lhuissier \& Villermaux (J. Fluid Mech., this issue, vol. 696, 2012, pp. 5-44) study and characterize the bursting of an emerging bubble. They show that this single type of event can, by itself, generate the droplet size distributions in a sea spray. This is a remarkable result showing how, in a complex system, a statistical distribution can be entirely produced by the dynamics of one dominant phenomenon.

\section{Introduction}

Water is mainly extracted from the ocean by evaporation. This is one of the main phases of the Earth's water cycle. As the air currents transport the vapour to cooler regions it condenses to form droplets in mists, clouds and rain. But this is not the only way by which water can be extracted from the ocean. Not all airborne water droplets are fresh water due to condensation. The haze observed on the photograph next to the title has a different origin. On this dry Atlantic coast of South Africa where the surf is heavy, the water is white with bubbles. Conversely, the air appears hazy, due to the spray formed of seawater droplets that it contains. As these droplets, directly extracted from the sea, are transported away they evaporate, leaving in suspension small solid particles composed mainly of salt that can be transported far from the coast. They form an important fraction of the aerosols present in the atmosphere. Woodcock (1953) showed that $100 \mathrm{~km}$ inland their distribution was the same at all levels from 150 to $1500 \mathrm{~m}$ and was not very different from what it was over the sea at the same altitude. As first suggested by Aitken (1881) these sub-micrometre-sized airborne particles have a role in the water cycle. Together with the other solid particles present in the atmosphere (e.g. dust, clay, or soot) they provide the nuclei needed for the condensation of water vapour into the liquid phase and thus for rain. Apart from salt, these droplets can transport surfactant molecules or pollutants (Blanchard 1964). They can also transfer bacteria extracted from the sea surface (Blanchard 1964; Blanchard \& Syzdek 1970). Recent measurements have shown that the density of these bacteria is seasonally modulated, with maxima during winter, probably because

$\dagger$ Email address for correspondence: couder@lps.ens.fr 
of a more efficient transfer from the sea due to stronger wind (Fahlgren et al. 2010). Finally the droplets were shown to contribute to the flux of electric charge between the sea and the atmosphere (see e.g. Aliverti \& Lovera 1939; Blanchard 1964).

\section{Overview}

The direct extraction of very small droplets from the sea is not an easy process because surface tension opposes it. Aliverti \& Lovera (1939) were probably the first to suggest that their production was directly related to the bursting of bubbles at the sea surface. Several phenomena can contribute to the formation of these bubbles, including wave breaking, rain, snow or emission by marine life. Because of their geophysical importance, many laboratory experiments in the last sixty years (e.g. Mason 1954) were devoted to the investigation of bubbles rising in water and bursting when they reach the surface. From these experiments it became clear that two distinct phenomena generate droplets. When a very small bubble breaks, the collapse of the cavity generates a high-speed jet rising from its bottom. This jet ejects one or a few droplets. For larger bubbles it is the bursting of their liquid film that is responsible for the production of a much larger number of droplets.

Using a simple laboratory experiment and making good use of the recent progress in fast video-cameras, Lhuissier \& Villermaux (2012) revisited the formation of the droplets in the second case. As shown in figure 1, the sequence of phenomena leading to the fragmentation of a bulk liquid into droplets proceeds by successive destabilizations in which the fluid dimensionality decreases step by step. The threedimensional bulk water is first stretched into a two-dimensional liquid hemispherical film that bursts into one-dimensional ligaments, the latter destabilizing into almost zero-dimensional droplets. As the air bubbles rise to the surface and reach it, their buoyancy provides the energy to form a hemispherical cap of radius $R$ and uniform thickness $h_{b}$. With time, the film thins, then breaks. It is well known that the phenomenon of marginal regeneration is responsible for the thinning of soap films stretched on a frame. Here it is shown to take place near the meniscus of negative curvature that connects the bubble to the water surface. The time evolution of the resulting thinning is measured and a new analysis of this phenomenon is proposed. It is based on the idea that Bénard-Marangoni convection is triggered at the bottom of the bubble. This idea leads to a good prediction of the observed thinning, confirmed by the observation of the periodicity of the convective plumes.

Then the film bursts. The puncture is observed to be initiated at the bottom of the hemisphere, in the convection region located above the bordering meniscus. This is also observed in experiments performed with a flat film. The measurements performed on the film thickness at the time of bursting are in good agreement with those obtained previously in other works. Lhuissier \& Villermaux (2012) proceed to a critical consideration of several phenomena to which the initial puncture could be ascribed: thermally activated noise; or turbulence in the marginal regeneration region. They show convincingly that it cannot be accounted for by the existing models of thickness fluctuations. This question of the nucleation of holes in relatively thick films remains open.

The hole opens at a constant tangential velocity $V=\sqrt{2 \sigma /\left(\rho h_{b}\right)}$ (where $\sigma$ is the surface tension, $\rho$ is the liquid density and $h_{b}$ is the film thickness) that results from the balance of inertia with surface tension forces as the liquid collects within the rim. The centripetal acceleration $\gamma=V^{2} / R$ exerted on the rim perpendicular to the shell surface of the bubble of radius $R$ induces its Rayleigh-Taylor destabilization 


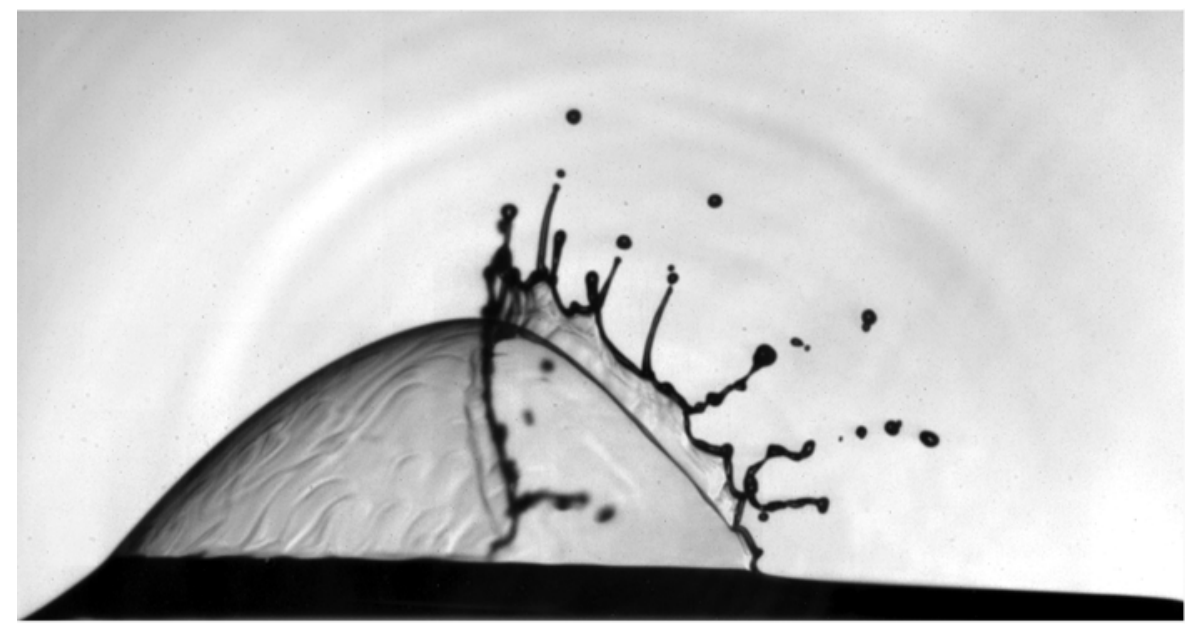

FIGURE 1. The breaking of an emerging bubble (courtesy of H. Lhuissier \& E. Villermaux).

with a wavelength $\lambda_{\perp} \simeq \sqrt{\sigma /(\rho \gamma)}$ that is the geometrical mean of the only two length scales (i.e. the bubble radius and film thickness) characterizing the bubble: $\lambda_{\perp} \simeq \sqrt{R h_{b}}$. The rim then forms a series of parallel ligaments. These liquid threads break into droplets by a Plateau-Rayleigh instability. Taking into account the succession of these phenomena the authors obtain an excellent estimate of the mean size of the droplets: $\langle d\rangle \simeq R^{3 / 8} h_{b}^{5 / 8}$; and an estimate of the number of drops produced per bubble: $N \simeq(R / a)^{3}\left(R / h_{b}\right)^{7 / 8}$ where $a=\sqrt{\sigma /(\rho g)}$ is the capillary length, below which capillarity dominates gravity. The resulting experimental drop size distribution $P(d)$ is in excellent agreement with the field measurements of Preobrazhenskii (1973) and Wu, Murray \& Lai (1984).

\section{Future}

This result can be compared to a previous achievement of Villermaux \& Bossa (2009) concerning the polydispersity of raindrops. While it was generally considered that the raindrop size distribution resulted from a complex interplay of drop breaking and drop coalescence in a turbulent flow, Villermaux \& Bossa (2009) demonstrated that the exponential size distribution of the raindrops could be quantitatively understood from the breaking of isolated single drops. More generally, whenever a complex statistical behaviour is observed in nature, it is necessary to first explore all the phenomena that can produce it. The temptation is then to think that they all contribute simultaneously to explain, for instance, an observed probability distribution. Fortunately, this discouraging perspective is usually not the case: in most situations one single type of process dominates the statistics. Both the present work on sea spray and the previous one on raindrops are beautiful demonstrations that a single physical process can produce a multiscale probability distribution and that a complete and precise analysis of a simple model can provide an accurate interpretation of global field measurements. 
Aitken, J. 1881 Dust, fog, and clouds. Nature 23, 384-385.

Aliverti, G. \& LoverA, G. 1939 I phenomeni meteorologica sul oceano e il campo ellectrico terrestre. Atti. Real. Acad. Sci., Torino 74, 573-590.

BLANCHARD, D. C. 1964 Sea-to-air transport of surface active material. Science 146, 396-397.

Blanchard, D. C. \& SYZDEK, L. D. 1970 Mechanisms for the water-to-air transfer and concentration of bacteria. Science 170, 626-628.

Fahlgren, C., Hagström, Å, Nilsson, E. D. \& Zweifel, U.-L. 2010 Annual variations in the diversity, viability, and origin of airborne bacteria. Appl. Env. Microbio. 76, 3015-3025.

Lhuissier, H. \& Villermaux, E. 2012 Bursting bubble aerosols. J. Fluid Mech. 696, 5-44.

MASON, B. J. 1954 Bursting of air bubbles at the surface of sea waters. Nature 174, 470-471.

PREOBRAZHENSKII, L. 1973 Estimate of the content of spray-drops in the near-water layer of the atmosphere. Fluid Mech. Sov. Res. 2, 95-100.

VillermauX, E. \& BossA, B. 2009 Single-drop fragmentation determines size distribution of raindrops. Nature Phys. 5, 697-702.

Woodcock, A. H. 1953 Salt nuclei in marine air as a function of altitude and wind force. J. Meteorol. 10, 362-371.

Wu, J., Murray, J. \& LAI, R. 1984 Production and distributions of sea spray. J. Geophys. Res. 89, 8163-8169. 